\title{
Certificación de sistemas de gestión y acreditación de la calidad en la educación superior
}

\author{
Rosa Mayelin Guerra Bretaña ${ }^{1}$ \\ Delia Alina Acosta Chávez ${ }^{2}$ (D) \\ Nuria Dávila Fernández ${ }^{3}$ (D) \\ Natalia Correa Hincapié 4 (D) \\ María Beatriz Valencia Bonilla ${ }^{5}$ (D) \\ ${ }^{1}$ Universidad de La Habana (UH), Cuba; ${ }^{2}$ Universidad Técnica Manabí (UTM), Ecuador; ${ }^{3}$ Centro de Gestión \\ y Desarrollo de la Calidad (CGDC), Cuba; ${ }^{4}$ Instituto Tecnológico Metropolitano (ITM), Colombia; ${ }^{5}$ Universidad \\ Tecnológica de Pereira (UTP), Colombia
}

Resumen. Los modelos de aseguramiento de la calidad en la educación superior permiten acreditar las instituciones. También, se han implementado sistemas para la gestión de la calidad ISO 9001 y en 2018 aparece la ISO 21001, con requisitos de gestión específicos para organizaciones educativas. El objetivo de este artículo es analizar cómo se relacionan estos diferentes modelos. El estudio se fundamenta en los puntos de contacto y diferencias entre los tres modelos, basado en el análisis de las normas y de los sistemas para el aseguramiento de la calidad de la Educación Superior, en Colombia, Cuba y Ecuador. Se observa que la implementación de un sistema de gestión contribuye al logro de los objetivos institucionales y la satisfacción de las partes interesadas, asimismo, en él deben incorporarse los requisitos establecidos en los modelos de acreditación en la educación superior. Además, se evidencia la complementariedad de la certificación y la acreditación de la calidad y las sinergias que pueden originarse a partir de la implementación conjunta de los modelos de aseguramiento externo y gestión interna de la calidad en la educación superior. De esta forma las organizaciones pueden estar mejor preparadas para enfrentar situaciones de riesgo, como la originada por la pandemia y contribuir al cumplimiento de los Objetivos de Desarrollo Sostenible.

Palabras clave: acreditación; calidad; certificación; educación superior; pandemia.

\section{Certificação de sistemas de gestão e acreditação da qualidade no ensino superior}

Resumo. Os modelos de garantia da qualidade no ensino superior permitem acreditar as instituições. Além disso, foram implementados os sistemas de gestão da qualidade ISO 9001 e, em 2018, surge a ISO 21001, com requisitos de gestão específicos para organizações educacionais. O objetivo deste artigo é analisar como se relacionam esses diferentes modelos. O estudo fundamenta-se nos pontos de contato e nas diferenças entre os três modelos, com base na análise das normas e dos sistemas de garantia de qualidade da Educação Superior na Colômbia, Cuba e Equador. Observa-se que a implantação de um sistema de gestão contribui para alcançar os objetivos institucionais e a satisfação das partes interessadas, da mesma forma que nele devem ser incorporados os requisitos estabelecidos nos modelos de acreditação no ensino superior. Além disso, evidencia-se a complementaridade da certificação e acreditação da qualidade e as sinergias que podem advir da implementação conjunta dos modelos de garantia externa e gestão interna da qualidade no ensino superior. Dessa forma, as organizações podem estar melhor preparadas para enfrentar situações de risco, como a provocada pela pandemia, e contribuir para o cumprimento dos Objetivos de Desenvolvimento Sustentável. Palavras-chave: acreditação; qualidade; certificação; educação superior; pandemia.

\section{Management systems certification and accreditation of quality in higher education}

Abstract. Quality assurance models in higher education allow institutions to be accredited. Also, ISO 9001 quality management systems have been implemented and in 2018 ISO 21001 appears, with specific management requirements for educational organizations. The objective of this article is to analyze how these different models are related. The study is based on the points of contact and differences between the three models, based on the analysis of standards and systems for quality assurance of Higher Education, in Colombia, Cuba and Ecuador. It is observed that the implementation of a management system contributes to the achievement of the institutional objectives and the satisfaction of the interested parties, likewise, it must incorporate the requirements established in the accreditation models in higher education. In addition, the complementarity of quality certification and accreditation and the synergies that may arise from the joint implementation of the external assurance and internal quality management models in higher education is evidenced. In this way, organizations can be better prepared to face risk situations, such as that caused by the pandemic and contribute to the fulfillment of the Sustainable Development Goals.

Keywords: accreditation; quality; certification; higher education; pandemic. 


\section{Introducción}

La Agenda 2030, adoptada de manera unánime en las Naciones Unidas (2015), estableció los Objetivos de Desarrollo Sostenible (ODS) con los cuales se adopta un conjunto de propósitos globales para erradicar la pobreza, proteger el planeta y asegurar la prosperidad para todos. El Objetivo de Desarrollo Sostenible (ODS) No. 4 busca garantizar una educación inclusiva, equitativa y de calidad, y promover oportunidades de aprendizaje durante toda la vida para todos. A partir de este compromiso, las instituciones de educación superior (IES) han tomado este objetivo como marco propicio para trazar sus estrategias (UNESCO-IESALC, 2020). Se parte de reconocer que, la calidad en la educación superior se logra desde el interior de las instituciones, gestionando sus procesos y con un enfoque a la satisfacción de las crecientes necesidades de los estudiantes y otros beneficiarios, sobre la base de la excelencia académica, la equidad y la pertinencia social de los programas. Además, el reconocimiento externo de la calidad de una IES se alcanza por la certificación de sus sistemas de gestión de la calidad o mediante la acreditación otorgada por el ente nacional encargado de asegurar la calidad de la educación superior.

Los modelos sectoriales, para el aseguramiento de la calidad en la educación superior, permiten acreditar las instituciones y los programas educativos. Además, existen requisitos mínimos para la autorización de estos. Por otra parte, muchas IES han implementado la norma de la Organización Internacional de Normalización (ISO), o sus adopciones nacionales, ISO 9001 (Organización Internacional de Normalización, 2015b) para la gestión de la calidad. Además, en el 2018 fue aprobada por la ISO una nueva norma ISO 21001 (ISO, 2018) que, por primera vez, establece requisitos específicos de los sistemas de gestión para las organizaciones educativas (SGOE). Estos requisitos han sido establecidos mediante el consenso de los organismos nacionales de normalización y otras partes interesadas, y buscan contribuir a "una educación de calidad y la promoción de una formación para la construcción de sociedades más humanas, equitativas y sostenibles" (Icontec, 2021b, p. 1).

El pensamiento basado en riesgos, que constituye la base de las normas de sistemas de gestión, ha contribuido a que las organizaciones que los han implementado se encuentren más preparadas para enfrentar los desafíos originados por la pandemia de la COVID-19 en los dos últimos años (Rodríguez-Rojas, 2021).

Al existir diferentes modelos que permiten evidenciar la calidad en la educación superior, es necesario preguntarse ¿Cómo estos se relacionan entre sí? ¿Aqué modelo deberían responder las IES? El objetivo de este trabajo es analizar cómo se relacionan los modelos sectoriales de acreditación de la calidad, con los esquemas de certificación de sistemas de gestión, basados en las normas internacionales adoptadas en los países. Este análisis contribuye a la toma de decisiones por parte de los directivos de las IES respecto a qué modelos adoptar, a partir de sus ventajas y dificultades. Además, se esclarecen los puntos de contacto y las diferencias existentes entre ellos, sobre la base del análisis de las normas aplicables y de los sistemas para el aseguramiento de la calidad de la Educación Superior, existentes en Colombia, Cuba y Ecuador, así como el análisis documental de las experiencias publicadas sobre el tema de estudio. 


\section{El Aseguramiento de la Calidad de la Educación Superior}

En los países de Latinoamérica y el Caribe se han desarrollado procesos de evaluación y acreditación en la Educación Superior, y se ha consolidado la cooperación internacional en este campo mediante el establecimiento de proyectos, como la Red Iberoamericana para la Evaluación y la Acreditación de la Calidad de la Educación Superior (RIACES), creada en 2003. Esta red está integrada por una treintena de agencias acreditadoras públicas o privadas, con presencia en 20 países de América Latina, el Caribe y Europa, y siete organismos regionales e internacionales. Así mismo, varios de los entes encargados de los procesos de acreditación en Educación Superior de América Latina pertenecen a la Red Internacional de Agencias de Acreditación de la Calidad de la Educación Superior - INQAAHE (International Network for Quality Assurance Agencies in Higher Education), la cual es una asociación mundial de más de 300 organizaciones activas en la teoría y práctica del aseguramiento de la calidad en la educación superior.

En Cuba, los sistemas de evaluación externa de la calidad de las IES se han desarrollado por más de 20 años (Noda, 2017). Desde su creación en 1976, el Ministerio de Educación Superior (MES) realizó acciones de control y evaluación sobre las IES, por su carácter rector de este nivel de formación y porque todas las Universidades, al ser públicas, se subordinan a él. En el año 2000 se crea el Sistema Universitario de Programas de Acreditación (SUPRA) y se funda la Junta de Acreditación Nacional (JAN), iniciándose sus trabajos con el Sistema de Evaluación y Acreditación (SEA) de Programas de Maestría. En el año 2003 se incorporó el SEA de carreras universitarias, complementándose posteriormente con el de Programas de Doctorado y el Sistema de Evaluación Institucional (SEI). En el 2015 se completó el Sistema con el SEA de Especialidades de Posgrado.

En 2018 el SUPRA fue sustituido por el Sistema de Evaluación y Acreditación de la Educación Superior (SEAES), como único sistema autorizado para acreditar los programas e instituciones de la educación superior, integrado por los cinco Subsistemas ya mencionados (MES, 2018). EI SEAES es un sistema integral para el aseguramiento y la mejora continua de la calidad en las IES, que se sustenta en la autoevaluación, la evaluación externa y la acreditación. En todos los casos la evaluación externa se realiza a los programas o IES que poseen la condición de autorizados, otorgada por las instancias correspondientes en el país.

Para cada subsistema se cuenta con un Patrón de Calidad y una Guía de Evaluación (con variables, criterios e indicadores) Además, se han establecidos Comités Técnicos Evaluadores nacionales para cada tipo de evaluación. La JAN ha establecido las siguientes categorías superiores de acreditación: Calificado, Certificado y Excelencia.

Para la evaluación de las IES cubanas las variables son:

1. Contexto institucional y pertinencia integral.

2. Recursos humanos.

3. Formación de pregrado.

4. Formación de investigación y postgrado.

5. Infraestructura.

6. Impacto social. 
Para solicitar a la Secretaría Ejecutiva de la JAN una evaluación, las IES deben haber realizado al menos dos procesos de autoevaluación y contar como mínimo con el $60 \%$ de las carreras universitarias y el $60 \%$ de los programas de posgrado académico acreditables con categoría superior de acreditación de la calidad.

En Colombia, la Ley 30 de 1992, en su Artículo 53, crea el Sistema Nacional de Acreditación, con el objetivo de garantizar a la sociedad que las IES cumplen altos estándares de calidad (Congreso de Colombia, 1992). Además, en su Artículo 54 establece que el Sistema Nacional de Acreditación cuenta con un Consejo Nacional de Acreditación integrado, entre otros, por las comunidades académicas y científicas y depende del Consejo Nacional de Educación Superior (CESU), el cual define su reglamento, funciones e integración. El Ministerio de Educación Nacional (MEN) regula el Sistema de Aseguramiento de la Calidad (SAC) de la Educación Superior y lo define como:

[...] el conjunto de instituciones e instancias definidas por el marco normativo vigente, que se articulan por medio de políticas y procesos diseñados, con el propósito de asegurar la calidad de las instituciones y de sus programas. Este sistema promueve en las instituciones los procesos de autoevaluación, auto regulación y mejoramiento de sus labores formativas, académicas, docentes, culturales y de extensión, contribuyendo al avance y fortalecimiento su comunidad y sus resultados académicos, bajo principios de equidad, diversidad, inclusión y sostenibilidad. (MEN, 2019, p. 4-5)

EI SAC incluye tres componentes: la evaluación, la información y el fomento (MEN, 2020). Participan en él un conjunto de instituciones, lo que le confiere un carácter supra Ministerial. Entre ellas, el Consejo Nacional de Acreditación (CNA) tiene como principal función promover y ejecutar la política de acreditación adoptada por el Consejo Nacional de Educación Superior y coordinar los procesos de acreditación. Tanto la acreditación institucional, como la de programas académicos, son otorgadas por el Ministerio de Educación Nacional a partir de la recomendación emanada del CNA.

A través de sus dos niveles, el registro calificado y la acreditación en alta calidad de programas e instituciones, el Sistema de Aseguramiento de la Calidad de la Educación Superior genera confianza en la calidad alcanzada. Si bien el primer nivel es obligatorio, para permitir el funcionamiento de los programas e instituciones, el segundo nivel, la acreditación en alta calidad, es voluntario. La acreditación en alta calidad es un acto voluntario. La vigencia de la acreditación en alta calidad puede ser de seis, ocho o diez años, en dependencia del "grado de consolidación, sostenibilidad e impacto de la Institución o el Programa académico, el grado de madurez de los procesos y la manera en la que la Institución asume los resultados o logros en función del mejoramiento continuo" (Correa et al. 2021, p. 136).

El Acuerdo 02 de 2020 (MEN, 2020), por el cual se actualizó el Modelo de Acreditación enAlta Calidad, establece los lineamientos para los procesos de autoevaluación para las IES y los programas académicos. Este Modelo va más allá de la gestión y sus resultados y se evalúan los impactos y logros que alcanzan las instituciones. Los lineamientos del Modelo están compuestos por factores, características y aspectos a evaluar. Los factores se refieren a los procesos, productos e impactos resultantes del cumplimiento de los objetivos de la IES o del programa académico. Mediante las 
características se materializan los factores y reflejan la calidad de los procesos. Con los aspectos detallados a evaluar se mide el desempeño de la característica y se observan las tendencias en el comportamiento de esta.

Los factores a evaluar para la acreditación institucional en Colombia son:

1. Identidad institucional.

2. Gobierno institucional y transparencia.

3. Desarrollo, gestión y sostenibilidad institucional.

4. Mejoramiento continuo y autorregulación.

5. Estructura y procesos académicos.

6. Aportes de la investigación, la innovación, el desarrollo tecnológico y la creación

7. Impacto social.

8. Visibilidad nacional e internacional.

9. Bienestar institucional.

10. Comunidad de profesores.

11. Comunidad de estudiantes.

12. Comunidad de egresados. ha logrado:

El reconocimiento de la alta calidad de las IES en Colombia supone que se

Desarrollo y apropiación de una sólida cultura de autoevaluación y autorregulación orientada al mejoramiento continuo, soportada en un sistema interno de aseguramiento de la calidad que permita evidenciar los logros y el desarrollo permanente de labores formativas, académicas, docentes, científicas, culturales y de extensión. (MEN, 2020, p. 28)

En Colombia, para la acreditación de programas e instituciones, se debe cumplir con el siguiente proceso:

a) Apreciación de condiciones iniciales

b) Autoevaluación

c) Evaluación externa por pares académicos

d) Evaluación integral

e) Expedición del acto administrativo que concede la acreditación o formula recomendaciones a la institución

Una vez realizado el proceso, en el cual se define la favorabilidad, o no, por parte del CNA para la acreditación institucional, la misma es otorgada o se procede con la generación de recomendaciones para que la institución pueda realizar su proceso de mejoramiento.

Según información del Sistema Nacional de Información de la Educación Superior (SNIES), de octubre de 2021, en Colombia hay trecientas diecisiete instituciones de educación superior y 61 seccionales que hacen parte de algunas de estas sedes principales. Con acreditación en alta calidad se cuenta con cuarenta y tres sedes principales y diecisiete seccionales. De esta forma se cuenta con el $13.5 \%$ de las instituciones principales acreditadas por el CNA y con el $27.9 \%$ de seccionales del total de instituciones de educación superior en el país. Se encuentra además que el $30 \%$ cuentan con una acreditación a cuatro años, el 11.67\% a 5 años, el $31.67 \%$ a seis años, el $18.33 \%$ a 8 años y el $8.33 \%$ a 10 años. 
En Ecuador, el aseguramiento de la calidad en la Educación Superior, también incluye los procesos de autorización y la acreditación de la calidad. Las IES deben mejorar continuamente la calidad de sus procesos y resultados, cumpliendo con la Ley Orgánica Reformatoria a la Ley Orgánica de Educación Superior - LOR LOES(Asamblea Nacional de Ecuador, 2018), y otra normativa a ella vinculada, como la establecida por el Consejo de Educación Superior (CES), la Secretaría Nacional de Ciencia, Tecnología e Innovación (SENESCYT).

El Sistema de Aseguramiento de la Calidad de la Educación Superior se establece en Ecuador como el "marco en el que se articulan diversos procesos para que las instituciones que son parte del Sistema de Educación Superior trabajen articuladamente por la mejora continua de la calidad de la educación superior" (CACES, 2018, p. 11). En la Ley Orgánica de Educación Superior del año 2000 se estableció el Sistema Nacional de Evaluación y Acreditación de la Educación, bajo el mandato del Consejo Nacional de Evaluación y Acreditación (CONEA). En esta etapa el proceso de acreditación era voluntario, por lo que un porcentaje muy reducido de universidades, escuelas politécnicas e institutos tecnológicos superiores se había sometido a este proceso para el año 2008 (Orozco et al., 2020). Ante esta situación, en julio de 2008 la Asamblea Nacional Constituyente comanda al CONEA realizar un informe sobre el nivel de las IES del país, clasificándolas en cinco niveles del "A", como la máxima calidad, al "E", como nivel de calidad no aceptable. Como resultado, 11 IES fueron calificadas en la categoría A, nueve en la B, 13 en la C, nueve en D y 26 en la E, de estas últimas 14 fueran cerradas en el año 2012.

Con la creación del Consejo de Evaluación, Acreditación y Aseguramiento de la Calidad de la Educación Superior (CEAACES), se realizó un nuevo proceso obligatorio de evaluación de las 54 universidades y escuelas politécnicas existentes en el 2013, en el que fueron categorizadas en cuatro niveles: $9 \%$ en la categoría A, $31 \%$ en la B, $44 \%$ en la C y $15 \%$ en la D. El hecho de que el $59 \%$ de las universidades y escuelas politécnicas ecuatorianas se encontraban en las dos categorías de calidad inferiores indicaba que debía darse continuidad a los procesos de evaluación de estas instituciones, por lo que en septiembre de 2015 el CEAACES publicó una nueva "Propuesta de adaptación del Modelo de Evaluación Institucional de la Universidades y Escuelas Politécnicas 2013 al proceso de re-categorización de Universidades y Escuelas Politécnicas 2015" (CEAACES, 2015a).

Entre 2015 y 2017 se realizó una nueva evaluación obligatoria de las ocho universidades y escuelas politécnicas ubicadas en la categoría D y la recategorización voluntaria de 12, evaluadas en las categorías B y C. Como resultado, para el 2018 se contaba con 55 universidades y escuelas politécnicas acreditadas, ocho de ellas en la Categoría A, 28 en la B y 19 en la C. En ese momento, se lanzó un nuevo proceso evaluativo de la mano del recién creado Sistema Interinstitucional de Aseguramiento de la Calidad, el cual que tiene como principales actores al Consejo de Educación Superior (CES), al Consejo de Aseguramiento de la Calidad de la Educación Superior (CACES) y a las propias instituciones de educación superior. Se reconoce entonces que las universidades y escuelas politécnicas son las encargadas de gestionar la calidad educativa, a partir de la autoevaluación permanente del cumplimiento de sus 
planes estratégicos de desarrollo institucional (PEDI), los planes operativos anuales (POA) y de los estándares de acreditación establecidos en el Modelo de evaluación externa (CACES, 2019).

El último proceso de evaluación externa de las universidades y escuelas politécnicas de Ecuador tuvo lugar en el último trimestre del año 2019. A diferencia de los modelos anteriores, en esta evaluación el objetivo fue la acreditación de las IES, no su categorización. En este proceso se evaluaron 55 universidades y escuelas politécnicas, 22 sedes y extensiones universitarias. Los Comités de Evaluación Externa estuvieron integrados por al menos dos pares nacionales, un par internacional y un técnico del CACES acompañó a cada Comité evaluador.

En el Modelo de evaluación externa de universidades y escuelas politécnicas de Ecuador se incluyen 20 estándares repartidos en cuatro ejes:

- Función sustantiva Docencia(Profesorado y estudiantado)-siete estándares.

- Función sustantiva Investigación - cuatro estándares.

- Función sustantiva Vinculación con la Sociedad - tres estándares.

- Condiciones institucionales - seis estándares.

Para las tres funciones sustantivas los estándares abarcan tres dimensiones: Planificación, Ejecución y Resultados.

Como resultado de este proceso 52 instituciones de educación superior fueron acreditadas, ya que alcanzaron los parámetros de cumplimiento establecidos en el modelo de evaluación. Las tres instituciones que no alcanzaron la acreditación presentaron un plan de mejoramiento, que contará con el acompañamiento del CACES por un período de hasta dos años.

Los esfuerzos por elevar la calidad de la educación superior en Ecuador también incidieron en los Institutos Superiores Tecnológicos (IST) los que fueron evaluados en el 2014 (CEAACES, 2015b). Los resultados de la evaluación indicaron que los 2019 IST como media solo lograron el $40 \%$ del puntaje máximo establecido en los parámetros de la evaluación. Una nueva evaluación se realizó en el 2020, a partir de un modelo específico acorde a las características de estas instituciones (CACES, 2020), las cuales pasaron por un proceso de unificación y fortalecimiento de su gestión, para convertirse en universidades tecnológicas.

Según la Organización Internacional de Normalización, el aseguramiento de la calidad es la parte de la gestión de la calidad orientada a proporcionar confianza en que se cumplirán los requisitos de la calidad (ISO, 2015a). Por tanto, los modelos sectoriales de evaluación y acreditación de la calidad en la Educación Superior se ajustan a esta definición, al proporcionar información a la sociedad sobre la calidad de las instituciones objeto de evaluación, fomentar una cultura de la calidad universitaria y el enfoque a la mejora continua (MES, 2018); promover "la búsqueda del cambio cualitativo de las instituciones de educación superior, basada en una permanente autorreflexión del conjunto de las comunidades educativas, orientada al mejoramiento continuo de sus tres funciones sustantivas..." (CACES, 2018, p. 32); y garantizar a la sociedad que los programas académicos y las instituciones de educación superior acreditados ofrecen el servicio público de educación con altos niveles de calidad (MEN, 2020). 
Para garantizar la calidad, se requiere que la acreditación vaya más allá del cumplimiento de indicadores, objetivos y metas, y que sea un instrumento que permita "obtener información confiable que conduzca a la toma de decisiones que conlleven a mejorar la calidad educativa de las IES" (Martínez et al., 2017, p. 85).

\section{La certificación de sistemas de gestión en las IES}

La certificación es una de las actividades de evaluación de la conformidad establecidas por la ISO. Como concepto más general, la evaluación de la conformidad es una demostración de que se cumplen los requisitos especificados, definidos como necesidades o expectativas establecidas en documentos normativos. Incluye actividades tales como: la inspección, la validación, la verificación, la certificación y la acreditación. Estas dos últimas actividades son las de mayor interés para este trabajo.

Según la definición de la ISO (2020), la certificación es la emisión de una declaración de tercera parte (atestación), relativa a un objeto de evaluación de la conformidad, basada en una decisión de que se ha demostrado el cumplimiento de los requisitos especificados en un documento normativo. El análisis que se realiza en este trabajo, se circunscribe a la certificación de un sistema de gestión de la calidad, por la norma ISO 9001:2015, o un sistema de gestión para organizaciones educativas, según ISO 21001:2018.

La acreditación, por su parte, es la atestación de tercera parte relativa a un organismo de evaluación de la conformidad, que manifiesta la demostración formal de su competencia, su imparcialidad y su operación coherente al llevar a cabo actividades específicas de evaluación de la conformidad (ISO, 2020). Como puede observarse, el concepto de acreditación en los modelos sectoriales empleados en la Educación Superior difiere sustancialmente del establecido en el esquema de la ISO. Las certificaciones de los sistemas de gestión son otorgadas por las entidades autorizadas y de preferencia acreditadas por los organismos nacionales correspondientes.

En Cuba la Oficina Nacional de Normalización es la entidad designada oficialmente en el país como Órgano Nacional de Certificación (Sosa y Llerandi, 2019). A través de su Dirección de Certificación realiza esta actividad respecto a los sistemas normalizados de gestión con alcance nacional y multisectorial, para lo cual asume las prácticas y cumple los requisitos internacionalmente aprobados para los organismos que realizan auditoría y certificación de sistemas de gestión, según la norma ISO/IEC 17021-1:2015 (ISO, 2015c).

El Órgano Nacional de Certificación de Cuba está acreditado por el Instituto Nacional de Normalización de Chile y aplica un conjunto de principios, comunes para los organismos de certificación, que inspiran confianza, orientan y facilitan la toma de decisiones ante situaciones imprevistas. Estos principios están relacionados con:

- La imparcialidad y las prácticas para su cumplimiento.

- La competencia de las personas que participan en los esquemas de certificación.

- Las responsabilidades, deberes y la autoridad de la dirección, del personal de certificación y de los Comités involucrados.

- La transparencia.

- La confidencialidad de las decisiones. 
- La receptividad y respuesta oportuna a las quejas.

- El enfoque basado en los riesgos asociados a los servicios de evaluación de la conformidad en función de garantizar que estos se realicen de manera competente, y de forma tal que se asegure la coherencia y la imparcialidad.

En Colombia, la máxima entidad de certificación acreditada en el país es el Instituto Colombiano de Normas Técnicas y Certificación - ICONTEC. Sin embargo, las certificaciones ISO pueden ser otorgadas por otras entidades que hayan sido aprobadas por la Superintendencia de Industria y Comercio (Gestión Colombia, 2014), entre estas entidades están: SGS Colombia S.A., BVQI Colombia Ltda., International Certification and Training S.A. (IC \& T), Cotecna Certificadora Services Ltda., la Corporación Centro de Investigación y Desarrollo Tecnológico y QLCT, organismo certificador de sistemas de gestión radicado en la Universidad Tecnológica de Pereira, acreditado por el Organismo Nacional de Acreditación de Colombia (ONAC).

ICONTEC es miembro de IQNet, la Red Mundial de los principales Organismos de Certificación, por lo que la institución que recibe una certificación ICONTEC recibe también el certificado IQNet, con lo que adquiere un carácter global, al estar respaldado por los miembros de esta red. ICONTEC está acreditado por el ONAC para la certificación de sistemas de gestión de la Calidad ISO 9001, no así para la certificación por la norma ISO 21001, que comenzó en fecha reciente (ICONTEC, 2021a).

Otro tipo de certificación que se otorga en el sector educativo colombiano es el relacionado con el Sistema Nacional de la Formación para el Trabajo y el Desarrollo Humano. Este tipo de oferta educativa puede jugar un importante papel en el proceso de transformación productiva del país (MEN, 2017). La certificación de calidad de la formación para el trabajo es el acto mediante el cual un organismo de tercera parte verifica y avala el cumplimiento de las normas técnicas de calidad por parte de las instituciones y los programas. Las Instituciones de Educación para el Trabajo y el Desarrollo Humano pueden certificarse con la Norma Técnica Colombiana NTC 5555 y sus programas con las normas NTC 5580, NTC 5581, NTC 5663, NTC 5664, NTC 5665, NTC 5666, según les corresponda (MEN, 2021).

En Ecuador los organismos de certificación de Sistemas de Gestión de Calidad, acreditados por el Servicio de Acreditación Ecuatoriano (SAE), son: Bureau Veritas Ecuador S.A.; AENOR Ecuador; S.G.S Ecuador S.A.; Icontec International S.A.; Calidad Internacional de Certificaciones C.I.C; COTECNA Certificadora Services Limitada; CERGESTCALAmérica Certification Group S.A. (SAE, 2021). La acreditación otorgada por SAE otorga confianza en las certificaciones por la norma ISO 9001, al avalar la competencia técnica de estos organismos de certificación para que auditen y certifiquen los sistemas de calidad de los interesados.

La certificación de los sistemas de gestión es un acto netamente voluntario. Para ello, la IES realiza la solicitud a un organismo de certificación, el cual procede a una auditoría externa y la emisión de una certificación de tercera parte, si se evidencia la conformidad con los requisitos de la norma en cuestión. Durante la implementación, mantenimiento y mejora del Sistema de Gestión de la Calidad (SGC), la institución debe haber realizado varias auditorías internas, en las que se genera información acerca de si el SGC: 
a) es conforme con los requisitos propios de la organización y los de la norma de referencia;

b) se implementa y mantiene eficazmente.

Además, se requiere haber realizado al menos una revisión por la dirección para asegurarse de la conveniencia, adecuación, eficacia y alineación continuas del SGC con la dirección estratégica de la institución (ISO, 2015b).

Por su parte, la norma ISO 21001, Organizaciones educativas — Sistemas de gestión para organizaciones educativas - Requisitos con orientación para su uso (ISO, 2018), está orientada al sector educativo en cualquiera de sus niveles y modalidades, y puede ser aplicada a cualquier organización que utilice un programa curricular para apoyar el desarrollo de la competencia mediante la enseñanza, el aprendizaje y la investigación. Así mismo, la norma puede ser implementada en organizaciones pertenecientes a otras mayores cuyo negocio principal no sea la educación. Esta es la primera norma internacional específica para el sector educación, la cual proporciona una herramienta de gestión común para organizaciones que proveen productos y servicios educativos capaces de cumplir con los requisitos de los estudiantes y otros beneficiarios.

La propia norma ISO 21001 menciona los beneficios potenciales de la implantación de sistemas de gestión para organizaciones educativas (SGOE), entre ellos (ISO, 2018):

a) mejor alineación de objetivos y actividades con la política, misión y visión);

b) mayor responsabilidad social al proporcionar una educación de calidad inclusiva y equitativa para todos;

c) aprendizaje más personalizado y una respuesta eficaz para todos los estudiantes y, en particular, para los estudiantes con necesidades especiales de educación, estudiantes a distancia y oportunidades de formación continua;

d) procesos y herramientas de evaluación coherentes para demostrar e incrementar la eficacia y la eficiencia;

e) mayor credibilidad de la organización;

f) un medio que permite a las organizaciones educativas demostrar su compromiso con prácticas de gestión educativa eficaces;

g) una cultura para la mejora organizativa;

h) armonización de las normas regionales, nacionales, abiertas, particulares y de otro tipo de normas dentro de un marco de referencia internacional;

i) mayor participación de las partes interesadas;

j) estimulación de la excelencia y la innovación.

La norma ISO 21001:2018 cumple con la estructura de alto nivel establecida por las Directivas de ISO para todas sus normas de sistemas de gestión. Esto facilita que las organizaciones educativas que ya tienen implementado un SGC ISO 9001:2015 transiten fácilmente al SGOE. De hecho, ya existen algunas publicaciones con experiencias de este tipo (Anh et al., 2021; Guerra et al., 2020; Nhon, 2020; Quimi, 2019; Rivera y Tupac-Yupanqui, 2019). 


\section{Relaciones entre los procesos de acreditación y certificación de la calidad en la educación superior}

Como se ha mostrado, los procesos sectoriales de acreditación en la educación superior y la certificación por los esquemas ISO de evaluación de la conformidad con las normas de sistemas de gestión tienen características y requisitos diferentes. Se reconoce que la acreditación externa, si bien involucra al personal de las instituciones en una autoevaluación previa, no puede por sí sola mejorar la calidad en las IES, ya que esto solo puede realizarse desde el interior de las propias instituciones, aplicando los principios reconocidos y los paradigmas contemporáneos de gestión de la calidad (Guerra y Meizoso, 2019; Jaya y Guerra, 2017).

Además, la acreditación es un proceso que se realiza en un intervalo de 6 a 10 años, por lo que es necesario una gestión interna sistémica y sistemática que garantice la sostenibilidad de la calidad alcanzada y esto lo aportan los sistemas normalizados de gestión. "El enfoque sistémico que propone el modelo de gestión de la calidad ... recalca la interdependencia de los factores y las interacciones entre los procesos como aspecto crucial para la gestión, así como el seguimiento constante para buscar la mejora continua" (Pedraza, 2010, p. 28).

A partir de reconocer la necesidad de relacionar los requisitos académicos y los genéricos de gestión de la calidad, ICONTEC cuenta con un servicio de Evaluación por un Modelo de Gestión para el Sector Educación con el cual se pretende facilitar la realización de la autoevaluación institucional, ya que el instrumento tuvo como elementos de entrada los aspectos que se abordan por el Ministerio de Educación Nacional (ICONTEC, 2021c). Además, proporciona una vía de mejoramiento de los procesos misionales, directivos y de apoyo que participan en el cumplimiento de los lineamientos de la calidad educativa. De la revisión de la información publicada por las IES colombianas, se determinó que más del 60 \% de las que están acreditadas en alta calidad también cuentan con la certificación ISO 9001:2015. Sin embargo, para octubre de 2021, ninguna de las IES acreditadas está certificada por la ISO 21001:2018.

Para optimizar los procesos de calidad, se ha identificado la articulación existente entre el SGC ISO 9001, el SGOE ISO 21001 y los modelos sectoriales de acreditación de la calidad. Esto permite evitar la duplicidad de esfuerzos e información, todo ello a partir de un enfoque a procesos, la mejora continua y la alineación estratégica de los diferentes modelos, para satisfacer las necesidades y expectativas de todas las partes interesadas (Castro et al., 2014; García y Gómez; Guerra et al., 2020; López, 2019; Nhon, 2020; Pedraza, 2010; Quimi, 2019; Quintero, 2012; Rivera y Tupac-Yupanqui, 2019; Trujillo-Suárez y Pedraza-Nájar, 2019).

Para fundamentar el análisis que se realiza, se presenta una tabla resumen de los elementos incluidos en los modelos de acreditación de Cuba, Colombia y Ecuador y cómo estos se pueden relacionar con los requisitos de las normas ISO 9001 e ISO 21001 (Tabla 1). La numeración referida en las columnas guarda relación con los numerales propios de cada documento de modelo de acreditación establecido por cada país. El modelo de acreditación en Cuba se establece por seis variables, el de Ecuador por veinte estándares y el de Colombia por 12 Factores. Estos, se comparan con los numerales establecidos en la norma ISO 21001. De esta forma, se reconoce que existe coherencia y complementariedad entre el enfoque sistémico de las normas y 
la acreditación institucional en la educación superior, que fortalece la calidad educativa y la hace sostenible, a través de la autorregulación institucional y el aporte sistémico del enfoque a procesos (Bahamón, 2008; Pedraza, 2010).

Tabla 1. Relación entre los elementos para la acreditación y los requisitos de las normas de gestión

\begin{tabular}{|c|c|c|c|}
\hline $\begin{array}{l}\text { Patrón de } \\
\text { calidad JAN, } \\
\text { Cuba }\end{array}$ & $\begin{array}{l}\text { Modelo Evaluación, } \\
\text { CACES, Ecuador }\end{array}$ & $\begin{array}{l}\text { Lineamientos } \\
\text { CNA, Colombia }\end{array}$ & Normas ISO \\
\hline \multirow{19}{*}{$\begin{array}{l}\text { Variable } 1 . \\
\text { Contexto } \\
\text { institucional } \\
\text { y pertinen- } \\
\text { cia integral }\end{array}$} & $\begin{array}{l}\text { Estándar 15: Planificación } \\
\text { estratégica y operativa }\end{array}$ & $\begin{array}{l}\text { Factor } 1 . \text { Identi- } \\
\text { dad Institucional }\end{array}$ & $\begin{array}{l}\text { 4. Contexto de la organi- } \\
\text { zación }\end{array}$ \\
\hline & $\begin{array}{l}\text { Estándar 18: Gestión interna } \\
\text { de la calidad }\end{array}$ & & $\begin{array}{l}4.1 \text { Comprensión de la or- } \\
\text { ganización y de su contexto } \\
4.2 \text { Comprensión de las }\end{array}$ \\
\hline & \multirow{17}{*}{$\begin{array}{l}\text { Además, los estándares de la } \\
\text { dimensión Planificación para } \\
\text { las tres funciones sustantivas }\end{array}$} & & $\begin{array}{l}\text { necesidades y expectativas } \\
\text { de las partes interesadas }\end{array}$ \\
\hline & & & $\begin{array}{l}4.3 \text { Determinación del al- } \\
\text { cance del sistema de ges- } \\
\text { tión para organizaciones } \\
\text { educativas }\end{array}$ \\
\hline & & & $\begin{array}{l}4.4 \text { Sistema de gestión } \\
\text { (para organizaciones edu- } \\
\text { cativas) }\end{array}$ \\
\hline & & \multirow{4}{*}{$\begin{array}{l}\text { Factor } 2 . \text { Go- } \\
\text { bierno institu- } \\
\text { cional y transpa- } \\
\text { rencia. }\end{array}$} & 5. Liderazgo \\
\hline & & & 5.1 Liderazgo y compromiso \\
\hline & & & 5.2 Política \\
\hline & & & $\begin{array}{l}\text { 5.3 Roles, responsabili- } \\
\text { dades y autoridades en la } \\
\text { organización }\end{array}$ \\
\hline & & \multirow{10}{*}{$\begin{array}{l}\text { Factor 3. Desa- } \\
\text { rrollo, gestión y } \\
\text { sostenibilidad } \\
\text { institucional }\end{array}$} & $\begin{array}{l}\text { 6. Planificación } \\
6.1 \text { Acciones para abordar } \\
\text { riesgos y oportunidades }\end{array}$ \\
\hline & & & $\begin{array}{l}6.2 \text { Objetivos de la organi- } \\
\text { zación educativa y planifica- } \\
\text { ción para lograrlos }\end{array}$ \\
\hline & & & $\begin{array}{l}\text { 6.3 Planificación de los } \\
\text { cambios }\end{array}$ \\
\hline & & & 7. Apoyo \\
\hline & & & 7.4 Comunicación \\
\hline & & & $\begin{array}{l}7.5 \text { Información documen- } \\
\text { tada }\end{array}$ \\
\hline & & & 8. Operación \\
\hline & & & $\begin{array}{l}\text { 8.1 Planificación y control } \\
\text { operacional }\end{array}$ \\
\hline & & & $\begin{array}{l}\text { 8.2 Requisitos para los } \\
\text { productos y servicios (edu- } \\
\text { cativos) }\end{array}$ \\
\hline & & & $\begin{array}{l}8.4 \text { Control de los procesos, } \\
\text { productos y servicios sumi- } \\
\text { nistrados externamente. }\end{array}$ \\
\hline
\end{tabular}




\begin{tabular}{|c|c|c|c|}
\hline $\begin{array}{c}\text { Patrón de } \\
\text { calidad JAN, } \\
\text { Cuba }\end{array}$ & $\begin{array}{l}\text { Modelo Evaluación, } \\
\text { CACES, Ecuador }\end{array}$ & $\begin{array}{l}\text { Lineamientos } \\
\text { CNA, Colombia }\end{array}$ & Normas ISO \\
\hline $\begin{array}{l}\text { Variable } 2 . \\
\text { Recursos } \\
\text { humanos }\end{array}$ & $\begin{array}{l}\text { Estándar 1: Planificación de } \\
\text { los procesos del profesorado } \\
\text { Estándar 2: Ejecución de } \\
\text { procesos del profesorado } \\
\text { Estándar } 3 \text { (A y B): Titularidad } \\
\text { del profesorado } \\
\text { Estándar } 4 \text { (Ay B): Formación } \\
\text { del profesorado }\end{array}$ & $\begin{array}{l}\text { Factor } 10 . \text { Co- } \\
\text { munidad de } \\
\text { profesores. }\end{array}$ & $\begin{array}{l}\text { 7. Apoyo } \\
\text { 7.1.2 Personas } \\
\text { 7.2 Competencia; } \\
\text { 7.3Toma de conciencia }\end{array}$ \\
\hline \multirow[t]{3}{*}{$\begin{array}{l}\text { Variable } 3 . \\
\text { Formación } \\
\text { de pregrado }\end{array}$} & $\begin{array}{l}\text { Estándar 1: Planificación de } \\
\text { los procesos del profesorado } \\
\text { Estándar 5: Planificación de } \\
\text { los procesos del estudiantado } \\
\text { Estándar 6: Ejecución de los } \\
\text { procesos del estudiantado } \\
\text { Estándar 7: Titulación del } \\
\text { estudiantado } \\
\text { (Modelo educativo y Gestión } \\
\text { académica) }\end{array}$ & $\begin{array}{l}\text { Factor } 5 . \text { Estruc- } \\
\text { tura y procesos } \\
\text { académicos. }\end{array}$ & \multirow[t]{5}{*}{$\begin{array}{l}\text { 8. Operación } \\
\text { 8.3 Diseño y desarrollo de } \\
\text { los productos y servicios } \\
\text { educativos; } \\
\text { 8.5 Producción y provisión } \\
\text { del servicio educativo; } \\
\text { 8.6 Liberación de los pro- } \\
\text { ductos y servicios educa- } \\
\text { tivos; } \\
\text { 8.7 Control de las salidas } \\
\text { educativas no conformes. }\end{array}$} \\
\hline & $\begin{array}{l}\text { Estándar 5: Planificación de } \\
\text { los procesos del estudiantado } \\
\text { Estándar 6: Ejecución de los } \\
\text { procesos del estudiantado } \\
\text { Estándar 7: Titulación del } \\
\text { estudiantado }\end{array}$ & $\begin{array}{l}\text { Factor } 11 . \text { Co- } \\
\text { munidad de } \\
\text { estudiantes. }\end{array}$ & \\
\hline & $\begin{array}{l}\text { Estándar 19: Bienestar estu- } \\
\text { diantil } \\
\text { Estándar 20: Igualdad de } \\
\text { oportunidades }\end{array}$ & $\begin{array}{l}\text { Factor 9. Bien- } \\
\text { estar institucio- } \\
\text { nal. }\end{array}$ & \\
\hline $\begin{array}{l}\text { Variable } 4 . \\
\text { Formación } \\
\text { en inves- } \\
\text { tigación y } \\
\text { postgrado } \\
\text { Variable } 6 . \\
\text { Impacto } \\
\text { social }\end{array}$ & $\begin{array}{l}\text { Estándar 8: Planificación de } \\
\text { los procesos de investigación } \\
\text { Estándar 9: Ejecución de los } \\
\text { procesos de investigación } \\
\text { Estándar 10: Producción } \\
\text { académica y científica } \\
\text { Estándar 11: Publicación de } \\
\text { artículos en revistas indizadas }\end{array}$ & $\begin{array}{l}\text { Factor } 6 . \\
\text { Aportes de la } \\
\text { investigación, } \\
\text { la innovación, } \\
\text { el desarrollo } \\
\text { tecnológico y la } \\
\text { creación }\end{array}$ & \\
\hline $\begin{array}{l}\text { Variable } 1 . \\
\text { Contexto } \\
\text { institucional } \\
\text { y pertinen- } \\
\text { cia integral }\end{array}$ & $\begin{array}{l}\text { Estándar 12: Planificación de } \\
\text { los procesos de vinculación } \\
\text { con la sociedad } \\
\text { Estándar 13: Ejecución de los } \\
\text { procesos de vinculación con } \\
\text { la sociedad } \\
\text { Estándar 14: Resultados de } \\
\text { los procesos de vinculación } \\
\text { con la sociedad }\end{array}$ & $\begin{array}{l}\text { Factor } 7 . \text { Impac- } \\
\text { to social. } \\
\text { Factor } 12 \text {. Co- } \\
\text { munidad de } \\
\text { egresados. } \\
\text { Factor } 8 \text {. Visibi- } \\
\text { lidad nacional e } \\
\text { internacional. }\end{array}$ & \\
\hline
\end{tabular}




\begin{tabular}{|c|c|c|c|}
\hline $\begin{array}{c}\text { Patrón de } \\
\text { calidad JAN, } \\
\text { Cuba }\end{array}$ & $\begin{array}{l}\text { Modelo Evaluación, } \\
\text { CACES, Ecuador }\end{array}$ & $\begin{array}{l}\text { Lineamientos } \\
\text { CNA, Colombia }\end{array}$ & Normas ISO \\
\hline $\begin{array}{l}\text { Variable } 5 . \\
\text { Infraestruc- } \\
\text { tura }\end{array}$ & $\begin{array}{l}\text { Estándar 15: Planificación } \\
\text { estratégica y operativa } \\
\text { Estándar 16: Infraestructura y } \\
\text { equipamiento informático } \\
\text { Estándar 17: Bibliotecas }\end{array}$ & $\begin{array}{l}\text { Factor 3. Desa- } \\
\text { rrollo, gestión y } \\
\text { sostenibilidad }\end{array}$ & $\begin{array}{l}\text { 7. Apoyo } \\
\text { 7.1 Recursos } \\
\text { 7.1.3 Infraestructura } \\
\text { 7.1.4 Ambiente para la } \\
\text { operación de los procesos } \\
\text { (educativos) }\end{array}$ \\
\hline $\begin{array}{l}\text { Variable } 1 \\
\text { Contexto } \\
\text { institucional } \\
\text { y pertinen- } \\
\text { cia integral }\end{array}$ & $\begin{array}{l}\text { Estándar 18: Gestión interna } \\
\text { de la calidad }\end{array}$ & $\begin{array}{l}\text { Factor } 4 \text {. Mejo- } \\
\text { ramiento conti- } \\
\text { nuo y autorregu- } \\
\text { lación. }\end{array}$ & $\begin{array}{l}\text { 9. Evaluación del desem- } \\
\text { peño } \\
\text { 10. Mejora }\end{array}$ \\
\hline
\end{tabular}

La certificación por las normas ISO 9001 e ISO 21001, si bien es una actividad voluntaria, puede ser parte de las políticas nacionales para fortalecer la Infraestructura de la Calidad. En el caso de Colombia, el Consejo Nacional de Política Económica y Social (CONPES), encargado de establecer los Lineamientos para una Política Nacional de la Calidad, reconoce al Consejo Nacional de Acreditación (CNA) de la Educación Superior, como una de las entidades que realizan actividades de acreditación en el país, si bien esta actividad no se rige por los esquemas de acreditación de la ISO. Esto no es así en el caso de Cuba, donde la acreditación en la Educación Superior no está relacionada entre los elementos de la Infraestructura Nacional de la Calidad. En Ecuador, tampoco se observa una fuerte relación entre la calidad vista con el enfoque sectorial del CACES y la certificación de los sistemas normalizados de gestión, si bien algunas IES si han optado por implementarlos.

En las actuales condiciones de enfrentamiento a la pandemia por la COVID-19 cuyos negativos impactos en la educación superior son evidentes (Canaza-Choque, 2020), los sistemas normalizados de gestión implementados en las IES han propiciado el marco organizacional necesario para la gestión de los riesgos a la calidad, ambientales y a la seguridad y salud en el trabajo generados por la actual situación. Asimismo, han contribuido a la toma de decisiones organizativas para mantener la continuidad del servicio educativo, gestionando de manera integral los riesgos que inciden en su desarrollo y que van a mantener sus efectos adversos durante algún tiempo (Guerra et al., 2021b). Por otra parte, los procesos de acreditación externa han debido adaptarse a la situación existente garantizando su desarrollo en condiciones de distanciamiento físico, apoyados en el uso de las herramientas de la información y las comunicaciones (Noda et al., 2021).

Respecto a la contribución de los procesos analizados al logro de los ODS, es necesario reconocer, en primer lugar, que la Organización de las Naciones Unidas para la Educación, la Ciencia y la Cultura (UNESCO) y otros organismos regionales, como el Instituto Internacional de la UNESCO para la Educación Superior en América Latina y el Caribe (UNESCO-IELSAC) han desarrollado documentos programáticos para la realización del Objetivo de Desarrollo Sostenible No. 4 Garantizar una educación inclusiva y equitativa de calidad y promover oportunidades de aprendizaje permanente 
para todos. Estos pronunciamientos deben ser respaldados por los gobiernos y las IES. De esta forma, tanto los procesos de evaluación externa y acreditación, como los SGOE, tributan a estos propósitos.

De manera específica, en el ODS No. 4 se plantean las metas siguientes:

- Meta 4.3: De aquí a 2030, asegurar el acceso en condiciones de igualdad para todos los hombres y las mujeres a una formación técnica, profesional y superior de calidad, incluida la enseñanza universitaria

- Meta 4.4: De aquí a 2030, aumentar sustancialmente el número de jóvenes y adultos que tienen las competencias necesarias, en particular técnicas y profesionales, para acceder al empleo, el trabajo decente y el emprendimiento.

Si bien todos los ODS y metas están relacionados entre sí, se resaltan las Metas 4.3 y 4.4, por el papel fundamental que juegan las IES en su cumplimiento, lo cual debe ser garantizado, en primera instancia, desde su gestión. A estos efectos, la implementación del SGOE está orientado a principios que estimulan el cumplimiento de estas Metas y del ODS 4 en su conjunto, entre ellos:

- Enfoque a los estudiantes y otros beneficiarios, involucrando activamente a los estudiantes en su propio aprendizaje, teniendo en cuenta las necesidades de la comunidad y los propósitos estratégicos de la IES.

- Liderazgo visionario, como premisa para mejorar la capacidad de la IES y sus personas para alcanzar los resultados e impactos esperados en los estudiantes, la comunidad y la sociedad en su conjunto.

- Compromiso de las personas, basado en el reconocimiento, el empoderamiento y la competencia.

- Responsabilidad social, basada en que la IES sea responsable por los impactos de sus decisiones y actividades en la sociedad, la economía y el medio ambiente, todo ellos sobre la base de la transparencia y el comportamiento ético.

- Accesibilidad y equidad, el que se basa en que las IES garanticen que "el grupo de personas más amplio posible tenga acceso a sus productos y servicios educativos, sujetos a sus limitaciones y recursos. También necesitan garantizar que todos los estudiantes puedan usar y beneficiarse de esos productos y servicios de manera equitativa" (ISO, 2018, pág. 55).

- Conducta ética en educación, la que crea un ambiente profesional, donde todos son tratados de manera equitativa, evitándose los conflictos de intereses y aportando valor a la sociedad.

Como corolario, la gestión de las IES, basada en los principios mencionados, con un enfoque orientado a la calidad en el desempeño de las personas y la organización es la base para aportar al cumplimiento de los ODS desde la educación superior. De igual forma, los procesos de evaluación y acreditación externa que aseguran una calidad educativa orientada a la excelencia académica, la pertinencia social y la equidad contribuyen al cumplimiento de estos objetivos.

\section{Conclusiones}

Los sistemas de gestión en la educación superior pueden involucrar los factores académicos, dados en los modelos de acreditación institucional, y los requisitos genéricos de gestión de la calidad, plasmados en la norma ISO 9001. Esto debe hacerse 
de manera integral para el logro de los objetivos institucionales y la satisfacción de las partes interesadas. Además, en la actualidad, al contar con la norma ISO 21001 ambos enfoques se alinean en el Sistema de Gestión para Organizaciones Educativas, aportando la complementariedad y sinergias para el logro de la certificación por los esquemas ISO de evaluación de la conformidad y la acreditación establecida como parte de los modelos de aseguramiento de la calidad educativa.

La certificación por la norma ISO 21001 puede servir de apoyo para las organizaciones encargadas de garantizar la calidad educativa. En el caso de la educación superior, esta certificación debe crear sinergias con los modelos de acreditación institucional, para garantizar y evidenciar el logro de niveles superiores de calidad educativa. Por otra parte, la certificación por un organismo acreditado es una garantía de la imparcialidad del proceso al no ser realizado por un organismo directamente interesado en la calidad de las IES. En Colombia se cuenta con esquemas de certificación por la norma ISO 21001, aunque no están acreditados aún. Estos esquemas deben ser desarrollados en Cuba y Ecuador para potenciar una certificación de tercera parte que aporte mayor imparcialidad a los esquemas sectoriales de acreditación de la calidad educativa.

En la actual situación de enfrentamiento a la COVID-19, los sistemas normalizados de gestión contribuyen a que las organizaciones estén mejor preparadas para enfrentar situaciones de riesgo, y puedan mantener la continuidad de los procesos educativos con calidad y seguridad. Finalmente, se evidencia cómo las IES desde su gestión y la participación en los procesos de evaluación externa y acreditación pueden contribuir al logro de los Objetivos del Desarrollo Sostenible en su conjunto y, de manera particular, al ODS No. 4.

\section{Referencias}

Anh, T. V., Linh, N. T., Nguyen, H. T. y Duan, T. C. (2021). ISO Standard Application in University Management Model: A Case Study. International Journal of Information and Education Technology, 194-199.

Asamblea Nacional de Ecuador (2018). Ley Orgánica de Educación Superior. Quito.

Bahamón, A. (2008). Incidencia del sistema de gestión de calidad en el proceso de acreditación. Educación y Desarrollo Social, 2(2), 36-41.

CACES. (2018). Política de Evaluación Institucional de Universidades y Escuelas Politécnicas en el marco del Sistema de Aseguramiento de la Calidad de la Educación Superior. Quito: Consejo de Aseguramiento de la Calidad de la Educación Superior.

CACES. (2019). Modelo de evaluación externa de universidades y escuelas politécnicas. Consejo de Aseguramiento de la Calidad de la Educación Superior. Recuperado de https://www. caces.gob.ec/institucional/

CACES. (2020). Modelo de evaluación institucional para los institutos superiores técnicos y tecnológicos en proceso de acreditación. Consejo de Aseguramiento de la Calidad de la Educación Superior. Recuperado de http://www.caces.gob.ec/879-2/

Canaza-Choque, F. A. (2020). Educación superior en la cuarentena global: disrupciones y transiciones. RIDU Revista Digital de Investigación en Docencia Universitaria, 14(2), 1-10. https://doi. org/10.19083/ridu.2020.1315 
Castro, A. J., Zapata, E. A., Jiménez, Y., Pizarro, A. M. y Marquéz, I. A. (2016). Sistema integrado de autoevaluación, acreditación y aseguramiento de la calidad - AAA, de la Institución Universitaria ITSA. Cartagena.

CEAACES. (2015a). Propuesta de adaptación del modelo de evaluación institucional de las universidades y escuelas politécnicas 2013 al proceso de recategorización de universidades y escuelas politécnicas. Ecuador.

CEAACES. (2015b). Informe de Evaluación de los Institutos Superiores Técnicos y Tecnológicos. Quito: Consejo de Evaluación, Acreditación y Aseguramiento de la Calidad de la Educación Superior (CEAACES).

Congreso de Colombia. (1992). Ley 30. Por la cual se organiza el servicio público de la Educación Superior. Bogotá.

Correa, N., Marín, Y. y Cornejo, J. W. (2021). Alta calidad en educación superior: reflexiones acerca del modelo colombiano de acreditación y sus posibles impactos. En J. J. Vizcaíno. Buenas prácticaspara el aseguramiento de la calidad en la educación superior (págs. 130-170). Latacunga: Universidad Técnica de Cotopaxi.

García, L. y Gómez, J. (2014). Alineación estratégica: factores del CNA, objetivos de calidad y la propuesta de valor. Caso: Facultad de Ciencias Económicas Universidad de San Buenaventura. Tesis de Maestría. Santiago de Cali: Universidad de San Buenaventura.

Gestión Colombia (2014). Entidades certificadoras . Recuperado de https://bit.ly/33WOSPi

Guerra, R. M. y Meizoso, M. C. (2019). Gestión de la calidad conceptos, modelos y herramientas (2da ed.). La Habana: Editorial Universidad de La Habana.

Guerra, R. M., Ramos, F. J. y Roque, R. (2020). Aplicación de la norma ISO 21001:2018 a la calidad de los programas de posgrado académico. Educación Médica Superior, 34(1), e2050.

Guerra, R. M., Sosa, R., Roque, R. y Ramos, F. J. (2021). Experiencias cubanas en la planificación de la continuidad del posgrado académico en tiempos de COVID-19. V Congreso Internacional de Investigación en Calidad. https://youtu.be/MpMQvvBO9P4

Icontec (2021a). Acreditaciones de nuestros servicios de Sistema de Gestión. Recuperado de https:// www.icontec.org/acreditaciones-servicios/

Icontec (2021b). Certificación ISO SG Organizaciones Educativas 21001. Recuperado de https://bit. Iy/3AhMbUI

Icontec (2021c). Evaluación Modelo de gestión para el sector Educación. Estándares educativos. Recuperado de https://bit.ly/3rwEfux

ISO (2015a). ISO 9000:2015 Sistemas de gestión de la calidad. Requisitos. Ginebra: Organización Internacional de Normalización.

ISO (2015b). ISO 9001:2015. Sistemas de Gestión de la Calidad. Requisitos. Ginebra: Organización Internacional de Normalización.

ISO (2015c). ISO/IEC 17021-1:2015 Evaluación de la conformidad. Requisitos para los organismos que realizan la auditoría y la certificación de sistemas de gestión. Parte 1. Requisitos. Ginebra: Organización Internacional de Normalización.

ISO (2018). ISO 21001:2018 Organizaciones educativas. Sistemas de gestión para organizaciones educativas. Requisitos con orientación para su uso. Ginebra: Organización Internacional de Normalización.

ISO (2020). ISO/IEC 17000:2020 Evaluación de la conformidad. Vocabulario y principios generales. Ginebra: Organización Internacional de Normalización.

Jaya Escobar, A. I. y Guerra Bretaña, R. M. (2017). El liderazgo y la participación como factores clave para la gestión de la calidad. Caso de la Universidad Estatal de Bolívar. Cofín Habana, 12(2), 206-225.

López, Ó. A. (2019). Análisis de la implementación del sistema de gestión de calidad ISO 9001:2015 y su articulación con la Acreditación de Alta Calidad, caso de estudio: Institución Universitaria Colegio Mayor del Cauca (IUCMC) y Fundación Universitaria de Popayán (FUP). Tesis de Maestría en Gestión de las Organizaciones. Popayán: Universidad Cooperativa de Colombia. 
Martínez, J. E., Tobón, S. y Romero, A. (2017). Problemáticas relacionadas con la acreditación de la calidad de la educación superior en América Latina. Innovación Educativa, 17(73), 79-96.

MEN (2017). Educación para el trabajo en el desarrollo humano. Ministerio de Educación Nacional. Recuperado de https://bit.ly/3llqS7f

MEN (2019). Decreto 1330 de 2019. Bogotá, Colombia. Recuperado de https://bit.ly/3GNCEGX

MEN (2020). Acuerdo 02 de 2020 Por el cual se actualiza el Modelo de Acreditación en Alta Calidad. Recuperado de https://bit.ly/3FKJHin

MEN (2021). Instituciones y Programas certificados en calidad. Ministerio de Educación Nacional. Recuperado de https://bit.ly/3GLafBF

MES (2018). Resolución No. 150/2018 Reglamento del Sistema de Evaluación y Acreditación de la Educación Superior (SEAES). La Habana: Ministerio de Educación Superior.

Naciones Unidas (2015). Transformar nuestro mundo: la Agenda 2030 para el desarrollo sostenible. A/ RES/70/1 Resolución 70/1 adoptada por laAsamblea General el 25 de septiembre de 2015.

Nhon, N. V. (2020). Integrated QMS ISO 9001 and EOMS ISO 21001 to help educational institutions on their continuous improvement path- Best practices. ANQ Congress 2020. Seoul.

Noda, M. E. (2017). Evaluación de la calidad y su acreditación en Cuba: actualidad y retos en el contexto de la Agenda Educativa 2030. En M. J. Lemaitre, Educación Superior y Sociedad. Aseguramiento de la CalidadenAmérica Latina (Vol. 22, págs. 87-110). Instituto Internacional de Unesco para la Educación Superior en América Latina y el Caribe (IESALC).

Noda, M. E., Ávila, J. C. y Hijuelos, N. J. (2021). Gestión de la calidad en la educación superior cubana. Actualidad y desafíos post COVID. Pedagogía 2021. Recuperado de https://bit.ly/3rC9S5Q.

Orozco, E. E., Jaya, A. I., Ramos, F. J. y Guerra, R. M. (2020). Retos a la gestión de la calidad en las instituciones de educación superior en Ecuador. Educación Médica Superior, 34(2), e2268.

Pedraza, X. L. (2010). Aporte del enfoque sistémico del modelo de gestión de la calidad NTC-ISO 9001 al modelo de acreditación institucional en instituciones privadas de educación superior. Signos, 2(2), 27-44. Recuperado de https://bit.ly/3rxvr7C

QLCT (2020). Organismo certificador QLCT. Recuperado de https://bit.ly/3tJX4gE

Quimi, D. I. (2019). Sistema de calidad enfocado a las normas ISO 9001 y 21001: caso Facultad de Ciencias Administrativas de la Universidad de Guayaquil. Universidad y Sociedad, 11(1), 279-288.

Quintero, O. E. (2012). Factores comunes de la acreditación de alta calidad y de la norma ISO 9001:2015:2008 en un sistema de gestión de calidad: caso Universidad de Medellín. Tesis MBA. Medellín: Universidad de Medellín.

Rivera, K. M., \& Tupac-Yupanqui, M. Á. (2019). La Norma ISO 21001:2018 - Apéndice normativo y su impacto en estudiantes de educación superior de Lima. INNOVA Research Journal, 4(3.2), 50-62.

Rodríguez-Rojas, Y. L. (2021). Sistemas de gestión normalizados y su contribución al control de los efectos organizacionales generados por la COVID-19. Signos, Investigación en Sistemas de Gestión, 13(2), 12-20. https://doi.org/10.15332/24631140.6661

SAE (2021). Organismos de Evaluación de la Conformidad Acreditados. Servicio de Acreditación Ecuatoriano. https://bit.ly/3tEWKzD

Sosa, R. y Llerandi, N. (2019). Evaluación de la conformidad acreditada y reconocida a nivel mundial. Caso Cuba. Memorias II Convención Internacional de Calidad "Habana 2019". 30 de septiembre al 3 de octubre de 2019 (págs. 507-516). La Habana: Palcograf.

Trujillo-Suárez, F. A. y Pedraza-Nájar, X. L. (2019). Articulación entre la NTC-ISO 9001:2015 y los lineamientos de acreditación de programas del CNA en Unitrópico. Signos. Investigación en Sistemas de Gestión, 11(2), 119-130.

UNESCO-IESALC (2020). Contribución de la educación superior a los objetivos de desarrollo sostenible: MarcoAnalítico. Caracas: Instituto Internacional de la UNESCO para la Educación Superior en América Latina y el Caribe.

\section{Cómo citar en APA:}

Guerra, R. M., Acosta, D. A., Dávila, N., Correa, N. y Valencia, M. B. (2022). Certificación de sistemas de gestión y acreditación de la calidad en la educación superior. Revista Iberoamericana de Educación, 88(1), 67-84. https://doi.org/10.35362/rie8814779 